\title{
Relativistic Symmetries of Hulthén Potential Incorporated with Generalized Tensor Interactions
}

\author{
A. N. Ikot, ${ }^{1}$ H. Hassanabadi, ${ }^{2}$ E. Maghsoodi, $^{2}$ and S. Zarrinkamar $^{3}$ \\ ${ }^{1}$ Theoretical Physics Group, Department of Physics, University of Uyo, Nigeria \\ ${ }^{2}$ Department of Basic Sciences, Shahrood Branch, Islamic Azad University, Shahrood, Iran \\ ${ }^{3}$ Department of Basic Sciences, Garmsar Branch, Islamic Azad University, Garmsar, Iran \\ Correspondence should be addressed to A. N. Ikot; ndemikotphysics@gmail.com
}

Received 5 May 2013; Accepted 2 August 2013

Academic Editor: Shi-Hai Dong

Copyright (C) 2013 A. N. Ikot et al. This is an open access article distributed under the Creative Commons Attribution License, which permits unrestricted use, distribution, and reproduction in any medium, provided the original work is properly cited.

Spin and pseudospin symmetries of the Dirac equation for a Hulthén potential with a novel tensor interaction, that is, a combination of the Coulomb and Yukawa potentials, are investigated using the Nikiforov-Uvarov method. The bound-state energy spectra and the radial wave functions are approximately obtained in the case of spin and pseudospin symmetries. The tensor interactions and the degeneracy-removing role are presented in details.

\section{Introduction}

The exact solutions of wave equations are still an interesting problem in fundamental quantum mechanics. Unfortunately, there are only a few potentials for which the Schrödinger, Dirac, Klein-Gordon, and Duffin-Kemmer-Petiau (DKP) equations can be exactly solved. Several potential models have been introduced to explore the relativistic and nonrelativistic energy spectra and the corresponding wave functions [1-5]. Jia et al. [6] have derived the bound-state solution of the Klein-Gordon equation under unequal scalar and vector kink-like potentials. By using the series expansion method, the authors in [7] have obtained the analytical solutions of the two-dimensional Schrödinger equation with the Morse potential. Pseudospin symmetry solution of Dirac equation for the modified Rosen-Morse potential is investigated in [8]. The DKP equation under a scalar Coulomb interaction is solved in [9] where the authors have used the ansatz approach. Ginocchio et al. [10-14] established the relationship between the pseudospin and the Dirac equation by recognizing that pseudo-orbital angular momentum $\widetilde{l}=l+1$ is nothing but the usual orbital angular momentum $l$ of the lower component of the Dirac spinor. They went further to show that, within the framework of the Dirac theory, the spin symmetry occurs when the difference of the potential between the repulsive Lorentz vector potential $V(r)$ and the attractive Lorentz scalar potential $S(r)$ is a constant; that is, $\Delta(r)=V(r)-S(r)=$ const. On the other hand, the pseudospin symmetry arises when the sum of the potentials is a constant; that is, $\Sigma(r)=V(r)+S(r)=$ const. Many researchers in the field have investigated the symmetries in the presence of various interactions. The list includes Manning-Rosen [15], Eckart [16], Hyllerass [17], Deng-Fan [18], Mobious square [19], Tietz [20], and Hyperbolical potentials [21]. The analysis of the symmetries has been done by using various methods such as Nikiforov-Uvarov (NU) technique [22], supersymmetric quantum mechanics (SUSYQM) [23], and many others [24, and references therein]. Due to the mathematical structure of the problem, the tensor interaction is often considered as a Coulomb or Cornell interaction [25]. Hassanabadi et al. were the first who introduced the Yukawa tensor interaction [26] besides the Coulomb-like term.

Here, we intend to report the solution of Dirac equation for the Hulthén potential under the generalized Coulomblike and Yukawa-like potentials as tensor interactions. The Hulthén potential is defined as $[27,28]$

$$
V(r)=-V_{0} \frac{e^{-2 \alpha r}}{\left(1-e^{-2 \alpha r}\right)},
$$




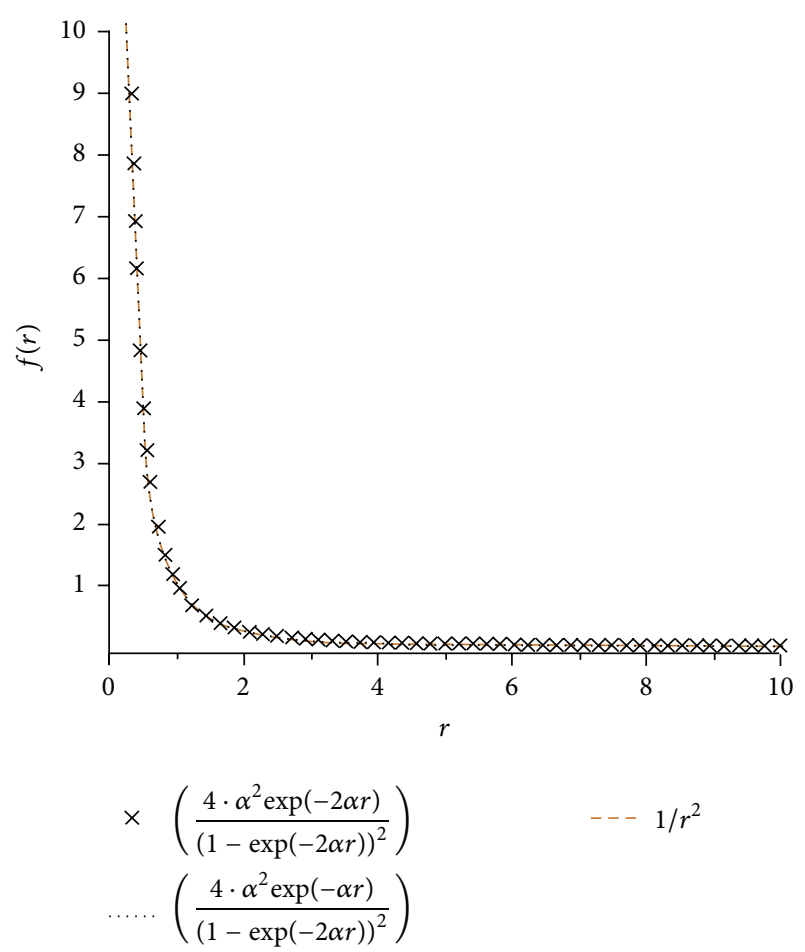

FIGURE 1: $1 / r^{2}$ and its approximations for $\alpha=0.05 \mathrm{fm}$.

where $V_{0}, \alpha$ are two positive real parameters and represent the strength and the screening range of the potential, respectively.

\section{Dirac Equation with a Tensor Interaction}

The Dirac equation under an attractive scalar potential $S(r)$, a repulsive vector potential $V(r)$, and a tensor potential $U(r)$ in the relativistic unit $(\hbar=c=1)$ is $[13,14]$

$$
\begin{aligned}
{[\vec{\alpha} \cdot \vec{p}} & +\beta(M+S(r))-i \beta \vec{\alpha} \cdot \vec{r} U(r)] \psi(r) \\
= & {[E-V(r)] \psi(r), }
\end{aligned}
$$

where $E$ is the relativistic energy of the system, $\vec{p}=-i \vec{\nabla}$ denotes the three-dimensional momentum operator, and $M$ stands for the mass of the fermionic particle. $\vec{\alpha}, \beta$ are the $4 \times 4$ Dirac matrices given as

$$
\vec{\alpha}=\left(\begin{array}{cc}
0 & \vec{\sigma}_{i} \\
\vec{\sigma}_{i} & 0
\end{array}\right), \quad \beta=\left(\begin{array}{cc}
I & 0 \\
0 & -I
\end{array}\right),
$$

where $I$ is $2 \times 2$ unitary matrix and $\vec{\sigma}_{i}$ are the Pauli three-vector matrices as follows:

$$
\sigma_{1}=\left(\begin{array}{ll}
0 & 1 \\
1 & 0
\end{array}\right), \quad \sigma_{2}=\left(\begin{array}{cc}
0 & -i \\
i & 0
\end{array}\right), \quad \sigma_{3}=\left(\begin{array}{cc}
1 & 0 \\
0 & -1
\end{array}\right) .
$$

The eigenvalues of the spin-orbit coupling operator are $\kappa=$ $(j+(1 / 2))>0, \kappa=-(j+(1 / 2))<0$ for unaligned $j=$ $l-(1 / 2)$ and the aligned $\operatorname{spin} j=l+(1 / 2)$, respectively. The set
$\left(H, K, J^{2}, J_{z}\right)$ forms a complete set of conserved quantities. Thus, we can write the spinors as

$$
\psi_{n \kappa}(r)=\frac{1}{r}\left(\begin{array}{c}
F_{n \kappa}(r) Y_{j m}^{l}(\theta, \varphi) \\
i G_{n \kappa}(r) Y_{j m}^{\tilde{l}}(\theta, \varphi)
\end{array}\right),
$$

where $F_{n \kappa}(r), G_{n \kappa}(r)$ represent the upper and lower components of the Dirac spinors. $Y_{j m}^{l}(\theta, \varphi), Y_{j m}^{\tau}(\theta, \varphi)$ are the spin and pseudospin spherical harmonics and $m$ is the projection on the $z$-axis. With other known identities,

$$
\begin{gathered}
(\vec{\sigma} \cdot \vec{A})(\vec{\sigma} \cdot \vec{B})=\vec{A} \cdot \vec{B}+i \vec{\sigma} \cdot(\vec{A} \times \vec{B}), \\
\vec{\sigma} \cdot \vec{p}=\vec{\sigma} \cdot \hat{r}\left(\vec{r} \cdot \vec{p}+i \frac{\vec{\sigma} \cdot \vec{L}}{r}\right)
\end{gathered}
$$

as well as

$$
\begin{gathered}
(\vec{\sigma} \cdot \vec{L}) Y_{j m}^{\tilde{l}}(\theta, \varphi)=(\kappa-1) Y_{j m}^{\widetilde{l}}(\theta, \varphi), \\
(\vec{\sigma} \cdot \vec{L}) Y_{j m}^{l}(\theta, \varphi)=-(\kappa+1) Y_{j m}^{l}(\theta, \varphi), \\
(\vec{\sigma} \cdot \widehat{r}) Y_{j m}^{l}(\theta, \varphi)=-Y_{j m}^{\tilde{l}}(\theta, \varphi), \\
(\vec{\sigma} \cdot \widehat{r}) Y_{j m}^{\widetilde{l}}(\theta, \varphi)=-Y_{j m}^{l}(\theta, \varphi),
\end{gathered}
$$

we find the following two coupled first-order Dirac equations:

$$
\begin{aligned}
& \left(\frac{d}{d r}+\frac{\kappa}{r}-U(r)\right) F_{n \kappa}(r)=\left(M+E_{n \kappa}-\Delta(r)\right) G_{n \kappa}(r), \\
& \left(\frac{d}{d r}-\frac{\kappa}{r}+U(r)\right) G_{n \kappa}(r)=\left(M-E_{n \kappa}+\Sigma(r)\right) F_{n \kappa}(r),
\end{aligned}
$$

where

$$
\begin{aligned}
& \Delta(r)=V(r)-S(r), \\
& \Sigma(r)=V(r)+S(r) .
\end{aligned}
$$

Decoupling the components, we obtain the second-order Schrödinger-like equation as

$$
\begin{aligned}
& \left\{\frac{d^{2}}{d r^{2}}-\frac{\kappa(\kappa+1)}{r^{2}}+\frac{2 \kappa U(r)}{r}-\frac{d U(r)}{d r}-U^{2}(r)\right. \\
& \quad-\left(M+E_{n \kappa}-\Delta(r)\right)\left(M-E_{n \kappa}+\Sigma(r)\right) \\
& \left.\quad+\left(\frac{d \Delta(r)}{d r}\left(\frac{d}{d r}+\frac{\kappa}{r}-U(r)\right)\right)\left(M+E_{n \kappa}-\Delta(r)\right)^{-1}\right\} \\
& \quad \times F_{n \kappa}(r)=0,
\end{aligned}
$$

$$
\begin{aligned}
& \left\{\frac{d^{2}}{d r^{2}}-\frac{\kappa(\kappa-1)}{r^{2}}+\frac{2 \kappa U(r)}{r}+\frac{d U(r)}{d r}-U^{2}(r)\right. \\
& \quad-\left(M+E_{n \kappa}-\Delta(r)\right)\left(M-E_{n \kappa}+\Sigma(r)\right) \\
& \left.-\left(\frac{d \Sigma(r)}{d r}\left(\frac{d}{d r}-\frac{\kappa}{r}+U(r)\right)\right)\left(M-E_{n \kappa}+\Sigma(r)\right)^{-1}\right\} \\
& \quad \times G_{n \kappa}(r)=0,
\end{aligned}
$$

where $\kappa(\kappa-1)=\tilde{l}(\widetilde{l}+1), \kappa(\kappa+1)=l(l+1)$. 
TABLE 1: Bound state for the pseudospin symmetry $\alpha=0.05 \mathrm{fm}, M=5 \mathrm{fm}^{-1}, V_{0}=4 \mathrm{fm}^{-1}$, and $C_{\mathrm{ps}}=-5 \mathrm{fm}^{-1}$.

\begin{tabular}{|c|c|c|c|c|c|c|c|}
\hline$\tilde{\ell} n, \kappa<0$ & $(\ell, j)$ & $\begin{array}{c}E_{n \kappa}^{\mathrm{ps}}\left(\mathrm{fm}^{-1}\right)=\text { Ref. [27] } \\
\left(V_{1}=0, H=0\right)\end{array}$ & $\begin{array}{c}E_{n \kappa}^{\mathrm{ps}}\left(\mathrm{fm}^{-1}\right) \\
\left(V_{1}=0.5, H=0.5\right)\end{array}$ & $n-1, \kappa>0$ & $(\ell+2, j+1)$ & $\begin{array}{c}E_{n \kappa}^{\mathrm{ps}}\left(\mathrm{fm}^{-1}\right)=\text { Ref. [27] } \\
\left(V_{1}=0, H=0\right)\end{array}$ & $\begin{array}{c}E_{n \kappa}^{\mathrm{ps}}\left(\mathrm{fm}^{-1}\right) \\
\left(V_{1}=0.5, H=0.5\right)\end{array}$ \\
\hline $1 \quad 1,-1$ & $1 S_{1 / 2}$ & $\begin{array}{l}-0.00862755 \\
-0.05738712\end{array}$ & $\begin{array}{l}-0.00255933 \\
-0.02961889\end{array}$ & 0,2 & $0 d_{3 / 2}$ & $\begin{array}{l}-0.00862755 \\
-0.05738712\end{array}$ & $\begin{array}{c}-0.01389734 \\
-0.10389112\end{array}$ \\
\hline $21,-2$ & $1 P_{3 / 2}$ & $\begin{array}{l}-0.01538462 \\
-0.10000000\end{array}$ & $\begin{array}{l}-0.00721029 \\
-0.06124936\end{array}$ & 0,3 & $0 f_{5 / 2}$ & $\begin{array}{l}-0.01538462 \\
-0.10000000\end{array}$ & $\begin{array}{l}-0.02260173 \\
-0.15622180\end{array}$ \\
\hline $31,-3$ & $1 d_{5 / 2}$ & $\begin{array}{l}-0.02413384 \\
-0.15233675\end{array}$ & $\begin{array}{l}-0.01389734 \\
-0.10389112\end{array}$ & 0,4 & $0 g_{7 / 2}$ & $\begin{array}{l}-0.02413384 \\
-0.15233675\end{array}$ & $\begin{array}{l}-0.03335506 \\
-0.21664494\end{array}$ \\
\hline $4 \quad 1,-4$ & $1 f_{7 / 2}$ & $\begin{array}{c}-0.03492388 \\
-0.21278254\end{array}$ & $\begin{array}{l}-0.02260173 \\
-0.15622180\end{array}$ & 0,5 & $0 h_{9 / 2}$ & $\begin{array}{c}-0.03492388 \\
-0.21278254\end{array}$ & $\begin{array}{l}-0.04621304 \\
-0.28340834\end{array}$ \\
\hline $1 \quad 2,-1$ & $2 S_{1 / 2}$ & $\begin{array}{l}-0.01538462 \\
-0.10000000\end{array}$ & $\begin{array}{l}-0.00721029 \\
-0.06124936\end{array}$ & 1,2 & $1 d_{3 / 2}$ & $\begin{array}{l}-0.01538462 \\
-0.10000000\end{array}$ & $\begin{array}{l}-0.02260173 \\
-0.15622180\end{array}$ \\
\hline $22,-2$ & $2 P_{3 / 2}$ & $\begin{array}{l}-0.02413384 \\
-0.15233675\end{array}$ & $\begin{array}{c}-0.01389734 \\
-0.10389112\end{array}$ & 1,3 & $1 f_{5 / 2}$ & $\begin{array}{l}-0.02413384 \\
-0.15233675\end{array}$ & $\begin{array}{l}-0.03335506 \\
-0.21664494\end{array}$ \\
\hline $32,-3$ & $2 d_{5 / 2}$ & $\begin{array}{l}-0.03492388 \\
-0.21278254\end{array}$ & $\begin{array}{l}-0.02260173 \\
-0.15622180\end{array}$ & 1,4 & $1 g_{7 / 2}$ & $\begin{array}{l}-0.03492388 \\
-0.21278254\end{array}$ & $\begin{array}{l}-0.04621304 \\
-0.28340834\end{array}$ \\
\hline $42,-4$ & $2 f_{7 / 2}$ & $\begin{array}{l}-0.04781723 \\
-0.27957698 \\
\end{array}$ & $\begin{array}{l}-0.03335506 \\
-0.21664494 \\
\end{array}$ & 1,5 & $1 h_{9 / 2}$ & $\begin{array}{l}-0.04781723 \\
-0.27957698 \\
\end{array}$ & $\begin{array}{l}-0.06125073 \\
-0.35469754\end{array}$ \\
\hline
\end{tabular}

TABLE 2: Bound state for the spin symmetry $\alpha=0.05 \mathrm{fm}, M=5 \mathrm{fm}^{-1}, V_{0}=4 \mathrm{fm}^{-1}$, and $C_{\mathrm{s}}=5 \mathrm{fm}^{-1}$.

\begin{tabular}{|c|c|c|c|c|c|c|c|c|}
\hline$\ell$ & $n, \kappa<0$ & $(\ell, j)$ & $\begin{array}{c}E_{n \kappa}^{\mathrm{s}}\left(\mathrm{fm}^{-1}\right)=\text { Ref. [27] } \\
\left(V_{1}=0, H=0\right)\end{array}$ & $\begin{array}{c}E_{n \kappa}^{s}\left(\mathrm{fm}^{-1}\right) \\
\left(V_{1}=0.5, H=0.5\right)\end{array}$ & $n, \kappa>0$ & $(\ell, j)$ & $\begin{array}{c}E_{n \kappa}^{\mathrm{s}}\left(\mathrm{fm}^{-1}\right)=\text { Ref. [27] } \\
\left(V_{1}=0, H=0\right)\end{array}$ & $\begin{array}{c}E_{n \kappa}^{\mathrm{s}}\left(\mathrm{fm}^{-1}\right) \\
\left(V_{1}=0.5, H=0.5\right)\end{array}$ \\
\hline 1 & $0,-2$ & $0 P_{3 / 2}$ & $\begin{array}{c}0.06784763 \\
0.00145930\end{array}$ & $\begin{array}{l}0.01709159 \\
0.00036476\end{array}$ & 0,1 & $0 P_{1 / 2}$ & $\begin{array}{c}0.06784763 \\
0.00145930\end{array}$ & $\begin{array}{l}0.15074992 \\
0.00328431\end{array}$ \\
\hline 2 & $0,-3$ & $0 d_{5 / 2}$ & $\begin{array}{c}0.15074992 \\
0.00328431\end{array}$ & $\begin{array}{c}0.06784763 \\
0.00145929\end{array}$ & 0,2 & $0 d_{3 / 2}$ & $\begin{array}{l}0.15074992 \\
0.00328431\end{array}$ & $\begin{array}{r}0.26338976 \\
0.00584101\end{array}$ \\
\hline 3 & $0,-4$ & $0 f_{7 / 2}$ & $\begin{array}{c}0.26338976 \\
0.00584101\end{array}$ & $\begin{array}{l}0.15074992 \\
0.00328431\end{array}$ & 0,3 & $0 f_{5 / 2}$ & $\begin{array}{c}0.26338976 \\
0.00584101\end{array}$ & $\begin{array}{c}0.40263365 \\
0.00913106\end{array}$ \\
\hline 4 & $0,-5$ & $0 g_{9 / 2}$ & $\begin{array}{c}0.40263365 \\
0.00913106\end{array}$ & $\begin{array}{c}0.26338976 \\
0.00584101\end{array}$ & 0,4 & $0 g_{7 / 2}$ & $\begin{array}{c}0.40263365 \\
0.00913106\end{array}$ & $\begin{array}{c}0.56482502 \\
0.01315663\end{array}$ \\
\hline 1 & $1,-2$ & $1 P_{3 / 2}$ & $\begin{array}{l}0.15074992 \\
0.00328431\end{array}$ & $\begin{array}{c}0.06784763 \\
0.00145929\end{array}$ & 1,1 & $1 P_{1 / 2}$ & $\begin{array}{l}0.15074992 \\
0.00328431\end{array}$ & $\begin{array}{l}0.26338976 \\
0.00584101\end{array}$ \\
\hline 2 & $1,-3$ & $1 d_{5 / 2}$ & $\begin{array}{c}0.26338976 \\
0.00584101\end{array}$ & $\begin{array}{l}0.15074992 \\
0.00328431\end{array}$ & 1,2 & $1 d_{3 / 2}$ & $\begin{array}{c}0.26338976 \\
0.00584101\end{array}$ & $\begin{array}{c}0.40263365 \\
0.00913106\end{array}$ \\
\hline 3 & $1,-4$ & $1 f_{7 / 2}$ & $\begin{array}{c}0.40263365 \\
0.00913106\end{array}$ & $\begin{array}{c}0.26338976 \\
0.00584101\end{array}$ & 1,3 & $1 f_{5 / 2}$ & $\begin{array}{c}0.40263365 \\
0.00913106\end{array}$ & $\begin{array}{c}0.56482502 \\
0.01315663\end{array}$ \\
\hline 4 & $1,-5$ & $1 g_{9 / 1}$ & $\begin{array}{c}0.56482502 \\
0.01315663\end{array}$ & $\begin{array}{c}0.40263365 \\
0.00913106\end{array}$ & 1,4 & $1 g_{7 / 2}$ & $\begin{array}{c}0.56482502 \\
0.01315663\end{array}$ & $\begin{array}{c}0.74599944 \\
0.01792038\end{array}$ \\
\hline
\end{tabular}

\section{Pseudospin Symmetry Limit}

The pseudospin symmetry limit occurs in Dirac equation when $d \Sigma(r) / d r=0$ or $\Sigma(r)=C_{\mathrm{ps}}=$ const. In this limit, we take $\Delta(r)$ as the Hulthén potential:

$$
\Delta(r)=-V_{0}\left(\frac{e^{-2 \alpha r}}{1-e^{-2 \alpha r}}\right)
$$

In addition, we propose a novel generalized tensor interaction of the form

$$
U(r)=U_{C}(r)+U_{Y}(r),
$$

where $U_{C}(r)$ and $U_{Y}(r)$ are the Coulomb-like and Yukawalike potentials defined as

$$
\begin{gathered}
U_{C}(r)=-\frac{H}{r}, \\
U_{Y}(r)=-V_{1} \frac{e^{-\alpha r}}{r}
\end{gathered}
$$

with

$$
H=\frac{z_{a} z_{b} e^{2}}{4 \pi \varepsilon_{0}},
$$

where $R_{e}$ is the Coulomb radius and $z_{a}$ and $z_{b}$ denote the charges of the projectile a and the target nuclei $b$, respectively. 

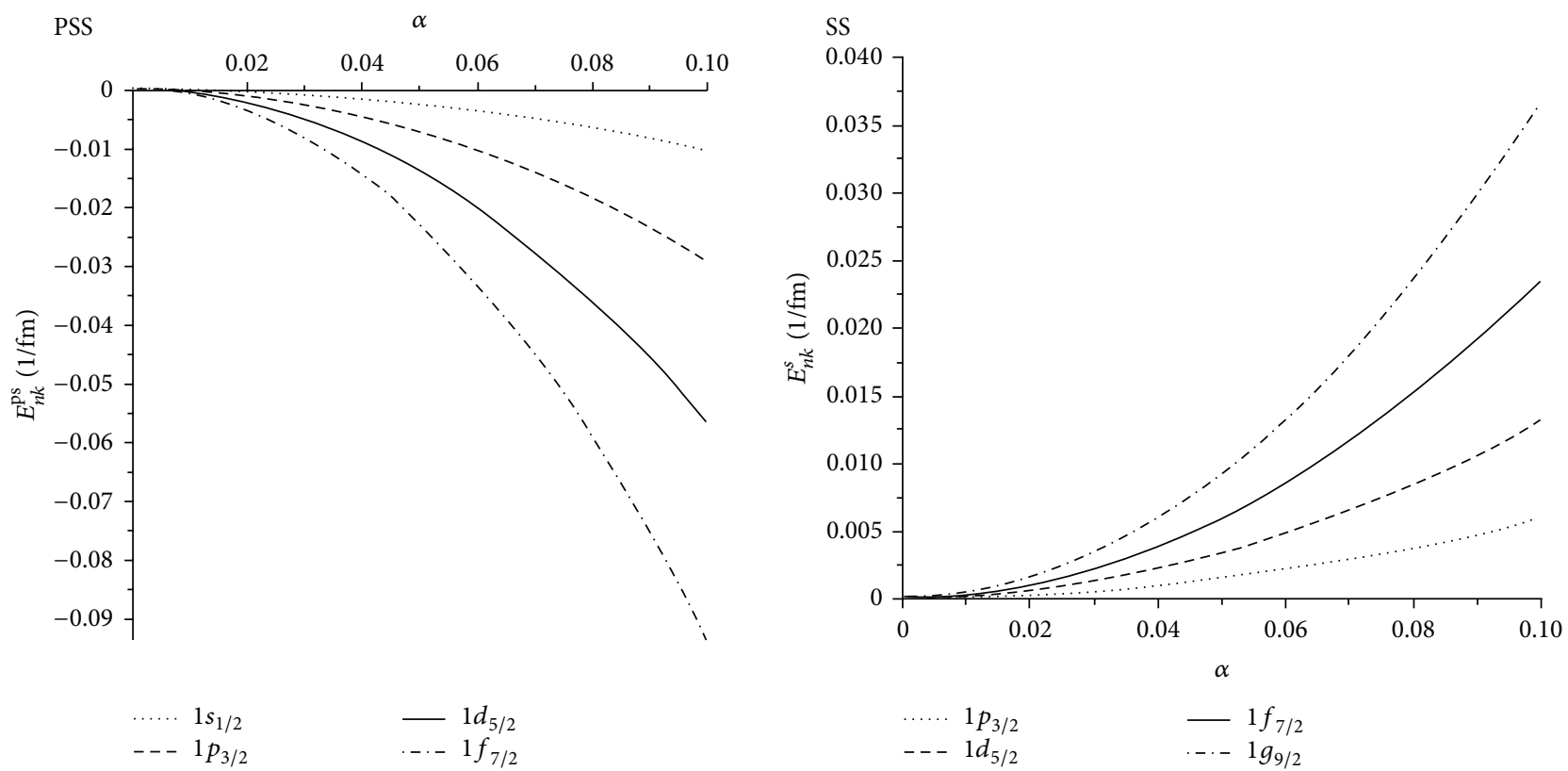

FIGURE 2: PSS: energy versus $\alpha$ for pseudospin symmetry limit for $V_{0}=4 \mathrm{fm}^{-1}, V_{1}=0.5 \mathrm{fm}^{-1}, H=0.5, M=5 \mathrm{fm}^{-1}$, and $C_{\mathrm{ps}}=-5 \mathrm{fm}^{-1}$. SS: energy versus $\alpha$ for spin symmetry limit for $V_{0}=4 \mathrm{fm}^{-1}, V_{1}=0.5 \mathrm{fm}^{-1}, H=0.5, M=5 \mathrm{fm}^{-1}$, and $C_{s}=5 \mathrm{fm}^{-1}$.

Also, $V_{1}$ is the depth of the potential. Substituting (14) into (13), we can write the tensor potential as

$$
U(r)=-\frac{1}{r}\left(H+V_{1} e^{-\alpha r}\right) .
$$

Substitution of (16) and (29) into (9) gives

$$
\begin{aligned}
& \left\{\frac{d^{2}}{d r^{2}}-\frac{\kappa(\kappa-1)}{r^{2}}-\frac{2 \kappa\left(H+V_{1} e^{-\alpha r}\right)}{r^{2}}+\frac{H}{r^{2}}+\frac{\alpha V_{1} e^{-\alpha r}}{r}\right. \\
& \quad+\frac{V_{1} e^{-\alpha r}}{r^{2}}-\frac{H^{2}}{r^{2}}-\frac{2 H V_{1} e^{-\alpha r}}{r^{2}}-\frac{V_{1}^{2} e^{-2 \alpha r}}{r^{2}}-\left(M+E_{n \kappa}^{\mathrm{ps}}\right) \\
& \left.\quad \times\left(M-E_{n \kappa}^{\mathrm{ps}}+C_{\mathrm{ps}}\right)-\left(M-E_{n \kappa}^{\mathrm{ps}}+C_{\mathrm{ps}}\right) V_{0} \frac{e^{-2 \alpha r}}{\left(1-e^{-2 \alpha r}\right)}\right\} \\
& \quad \times G_{n \kappa}^{\mathrm{ps}}(r)=0 .
\end{aligned}
$$

It is well known that the above equation cannot be exactly solved due to the centrifugal term $r^{-2}$. In order to get rid of the centrifugal term, we make use of the following approximation $[27,29]$ (see Figure 1):

$$
\frac{1}{r^{2}} \approx \frac{4 \alpha^{2} e^{-2 \alpha r}}{\left(1-e^{-2 \alpha r}\right)^{2}}, \quad \frac{1}{r^{2}} \approx \frac{4 \alpha^{2} e^{-\alpha r}}{\left(1-e^{-2 \alpha r}\right)^{2}},
$$

where $\kappa=-\widetilde{\ell}$ and $\kappa=\widetilde{\ell}+1$ for $\kappa<0$ and $\kappa>0$, respectively. Using a new variable of the form $s=e^{-2 \alpha r}$ and introducing

$$
\begin{gathered}
f_{1}^{\mathrm{ps}}=\frac{\varepsilon_{\mathrm{ps}}^{2}}{4 \alpha^{2}}-\frac{\beta_{\mathrm{ps}}}{4 \alpha^{2}}+V_{1}\left(V_{1}+\frac{1}{2}\right), \\
f_{2}^{\mathrm{ps}}=\frac{2 \varepsilon_{\mathrm{ps}}^{2}}{4 \alpha^{2}}-\frac{\beta_{\mathrm{ps}}}{4 \alpha^{2}}-V_{1}\left(2 H+2 \kappa-\frac{3}{2}\right)-\eta_{\kappa}\left(\eta_{\kappa}-1\right), \\
f_{3}^{\mathrm{ps}}=\frac{\varepsilon_{\mathrm{ps}}^{2}}{4 \alpha^{2}},
\end{gathered}
$$

where

$$
\begin{gathered}
\varepsilon_{\mathrm{ps}}^{2}=\left(M+E_{n \kappa}^{\mathrm{ps}}\right)\left(M-E_{n \kappa}^{\mathrm{ps}}+C_{\mathrm{ps}}\right), \\
\beta_{\mathrm{ps}}=\left(M-E_{n \kappa}^{\mathrm{ps}}+C_{\mathrm{ps}}\right) V_{0}, \\
\kappa(\kappa-1)+2 \kappa H-H+H^{2}=(\kappa+H)(\kappa+H-1) \\
=\eta_{\kappa}\left(\eta_{\kappa}-1\right) \longrightarrow \eta_{\kappa}=(\kappa+H),
\end{gathered}
$$

(17) is transformed to

$$
\frac{d^{2} G_{n \kappa}^{\mathrm{ps}}}{d s^{2}}+\frac{1}{s} \frac{d G_{n \kappa}^{\mathrm{ps}}}{d s}+\frac{-f_{1}^{\mathrm{ps}} s^{2}+f_{2}^{\mathrm{ps}} s-f_{3}^{\mathrm{ps}}}{s^{2}(1-s)^{2}} G_{n \kappa}^{\mathrm{ps}}=0 .
$$

Comparing (23) with (A.1), we find

$$
\begin{gathered}
\alpha_{1}=\alpha_{2}=\alpha_{3}=1, \quad \xi_{1}^{\mathrm{ps}}=f_{1}^{\mathrm{ps}}, \\
\xi_{2}^{\mathrm{ps}}=f_{2}^{\mathrm{ps}}, \quad \xi_{3}^{\mathrm{ps}}=f_{3}^{\mathrm{ps}} .
\end{gathered}
$$


From (A.4) one can determine the rest of coefficients as

$$
\begin{aligned}
& \alpha_{4}=0, \quad \alpha_{5}=-\frac{1}{2}, \quad \alpha_{6}=\frac{1}{4}+f_{1}^{\mathrm{ps}}, \\
& \alpha_{7}=-f_{2}^{\mathrm{ps}}, \quad \alpha_{8}=f_{3}^{\mathrm{ps}}, \quad \alpha_{9}=\frac{1}{4}+f_{1}^{\mathrm{ps}}+f_{3}^{\mathrm{ps}}-f_{2}^{\mathrm{ps}}, \\
& \alpha_{10}=1+2 \sqrt{f_{3}^{\mathrm{ps}}}, \\
& \alpha_{11}=2+2\left(\sqrt{\frac{1}{4}+f_{1}^{\mathrm{ps}}+f_{3}^{\mathrm{ps}}-f_{2}^{\mathrm{ps}}}+\sqrt{f_{3}^{\mathrm{ps}}}\right), \\
& \alpha_{12}=\sqrt{f_{3}^{\mathrm{ps}}}, \quad \\
& \alpha_{13}=-\frac{1}{2}-\left(\sqrt{\frac{1}{4}+f_{1}^{\mathrm{ps}}+f_{3}^{\mathrm{ps}}-f_{2}^{\mathrm{ps}}}+\sqrt{f_{3}^{\mathrm{ps}}}\right) .
\end{aligned}
$$

Substitution of the values of the parameters given by (25) into (A.2) and (A.3) gives us the following relations for the eigenfunctions and energy eigenvalues:

$$
\begin{aligned}
G_{n \kappa}^{\mathrm{ps}}(r)= & e^{-2 \alpha \sqrt{f_{3}^{\mathrm{ps}}} r}\left(1-e^{-2 \alpha r}\right)^{(1 / 2)+\sqrt{(1 / 4)+f_{1}^{\mathrm{ps}}+f_{3}^{\mathrm{ps}}-f_{2}^{\mathrm{ps}}}} \\
\times & P_{n}^{\left(2 \sqrt{f_{3}^{\mathrm{ps}}}, 2 \sqrt{(1 / 4)+f_{1}^{\mathrm{ps}}+f_{3}^{\mathrm{ps}}-f_{2}^{\mathrm{ps}}}\right)}\left(1-2 e^{-2 \alpha r}\right), \\
& \rho_{p}+\lambda_{p} \sqrt{f_{3}^{\mathrm{ps}}}+\frac{\beta_{p}}{4 \alpha^{2}}=0,
\end{aligned}
$$

where

$$
\begin{aligned}
\rho_{p}= & n^{2}+n+\frac{1}{2}+(2 n+1) \\
& \times \sqrt{\frac{1}{4}+\left(\kappa+V_{1}+H\right)\left(\kappa+V_{1}+H-1\right)} \\
& +\eta_{\kappa}\left(\eta_{\kappa}-1\right)+V_{1}\left(2 H+2 \kappa-\frac{3}{2}\right), \\
\lambda_{p}=2 n+1 & +2 \sqrt{\frac{1}{4}+\left(\kappa+V_{1}+H\right)\left(\kappa+V_{1}+H-1\right)},
\end{aligned}
$$

On the other hand, the upper component can be found by using the following relation:

$$
F_{n, \kappa}^{\mathrm{ps}}(r)=\frac{1}{M-E_{n \kappa}^{\mathrm{ps}}+C_{\mathrm{ps}}}\left(\frac{d}{d r}-\frac{\kappa}{r}+U(r)\right) G_{n \kappa}^{\mathrm{ps}}(r) .
$$

\section{Spin Symmetry Limit}

In this section, we consider the spin symmetry limit where $d \Delta(r) / d r=0$ or $\Delta(r)=C_{\mathrm{ps}}=$ const. As in the previous section, we consider

$$
\begin{aligned}
& \Sigma(r)=-V_{0}\left(\frac{e^{-2 \alpha r}}{1-e^{-2 \alpha r}}\right), \\
& U(r)=-\frac{1}{r}\left(H+V_{1} e^{-\alpha r}\right) .
\end{aligned}
$$

Substitution of (16) and (22) into (10) gives

$$
\begin{aligned}
& \left\{\frac{d^{2}}{d r^{2}}-\frac{\kappa(\kappa+1)}{r^{2}}-\frac{2 \kappa H}{r^{2}}-\frac{H^{2}}{r^{2}}-\frac{H}{r^{2}}-\frac{2 \kappa V_{1} e^{-\alpha r}}{r^{2}}\right. \\
& -\frac{\alpha V_{1} e^{-\alpha r}}{r}-\frac{V_{1} e^{-\alpha r}}{r^{2}}-\frac{2 H V_{1} e^{-\alpha r}}{r^{2}}-\frac{V_{1}^{2} e^{-2 \alpha r}}{r^{2}} \\
& \left.-\varepsilon_{s}^{2}+\frac{\beta_{s} e^{-2 \alpha r}}{\left(1-e^{-2 \alpha r}\right)}\right\} F_{n \kappa}^{s}(r)=0,
\end{aligned}
$$

with $\varepsilon_{s}^{2}=\left(E_{n \kappa}^{s}+M-C_{s}\right)\left(M-E_{n \kappa}^{s}\right), \beta_{s}=\left(E_{n \kappa}^{s}+M-C_{s}\right) V_{0}$, and $k=\ell$ and $k=-\ell-1$ for $k<0$ and $k>0$, respectively. Substituting (18) into (30), we have

$$
\begin{aligned}
& \left\{\frac{d}{d r^{2}}-\frac{4 \alpha^{2} \Lambda_{\kappa}\left(\Lambda_{\kappa}-1\right) e^{-2 \alpha r}}{\left(1-e^{-2 \alpha r}\right)^{2}}-\frac{8 \alpha^{2} \kappa V_{1} e^{-2 \alpha r}}{\left(1-e^{-2 \alpha r}\right)^{2}}-\frac{2 \alpha^{2} V_{1} e^{-2 \alpha r}}{\left(1-e^{-2 \alpha r}\right)}\right. \\
& -\frac{4 \alpha^{2} V_{1} e^{-2 \alpha r}}{\left(1-e^{-2 \alpha r}\right)^{2}}-\frac{8 \alpha^{2} H V_{1} e^{-2 \alpha r}}{\left(1-e^{-2 \alpha r}\right)^{2}}-\frac{4 \alpha^{2} V_{1}^{2} e^{-4 \alpha r}}{\left(1-e^{-2 \alpha r}\right)^{2}} \\
& \left.-\varepsilon_{s}^{2}+\frac{\beta_{s} e^{-2 \alpha r}}{\left(1-e^{-2 \alpha r}\right)}\right\} F_{n \kappa}^{s}(r)=0,
\end{aligned}
$$

where

$$
\begin{gathered}
\kappa(\kappa+1)+2 \kappa H+H+H^{2}=(\kappa+H+1)(\kappa+H) \\
=\Lambda_{\kappa}\left(\Lambda_{\kappa}-1\right) \longrightarrow \Lambda_{\kappa}=(\kappa+H+1) .
\end{gathered}
$$

Introducing a new transformation of the form $y=e^{-2 \alpha r}$, we arrive at the Schrödinger-like equation:

$$
\left\{\frac{d^{2}}{d y^{2}}+\frac{1}{y} \frac{d}{d y}+\frac{-f_{1}^{s} y^{2}+f_{2}^{s} y-f_{3}^{s}}{y^{2}(1-y)^{2}}\right\} F_{n \kappa}^{s}(y)=0,
$$

where

$$
\begin{aligned}
f_{1}^{s} & =\frac{\varepsilon_{s}^{2}}{4 \alpha^{2}}+\frac{\beta_{s}}{4 \alpha^{2}}+V_{1}\left(V_{1}-\frac{1}{2}\right), \\
f_{2}^{s} & =\frac{2 \varepsilon_{s}^{2}}{4 \alpha^{2}}+\frac{\beta_{s}}{4 \alpha^{2}}-\Lambda_{\kappa}\left(\Lambda_{\kappa}-1\right)-\left(2 \kappa+2 H+\frac{3}{2}\right) V_{1}, \\
f_{3}^{s} & =\frac{\varepsilon_{s}^{2}}{4 \alpha^{2}} .
\end{aligned}
$$

Using the same approach we used to solve (23), the energy equation is obtained as

$$
\rho_{s}+\lambda_{s} \sqrt{f_{3}^{s}}-\frac{\beta_{s}}{4 \alpha^{2}}=0,
$$




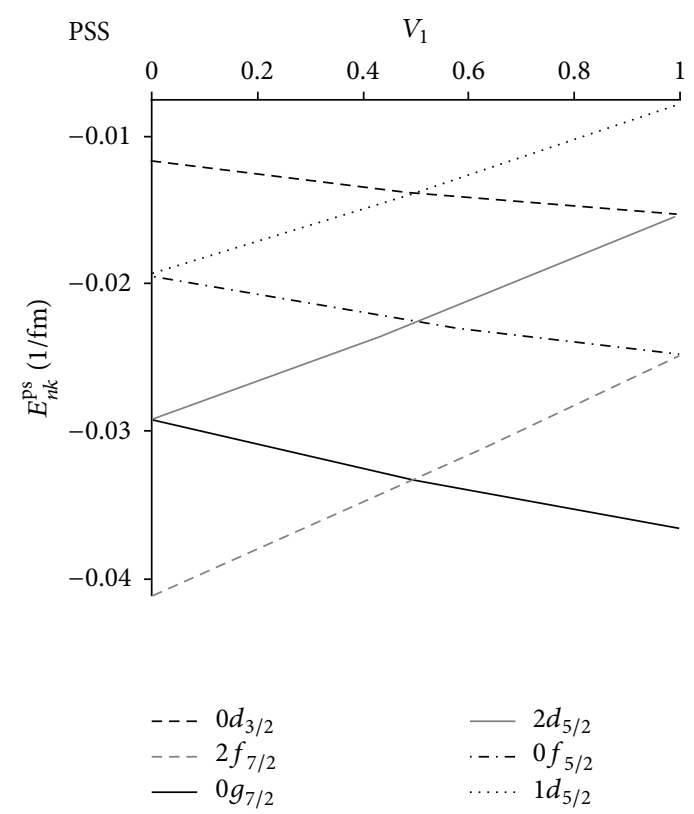

SS

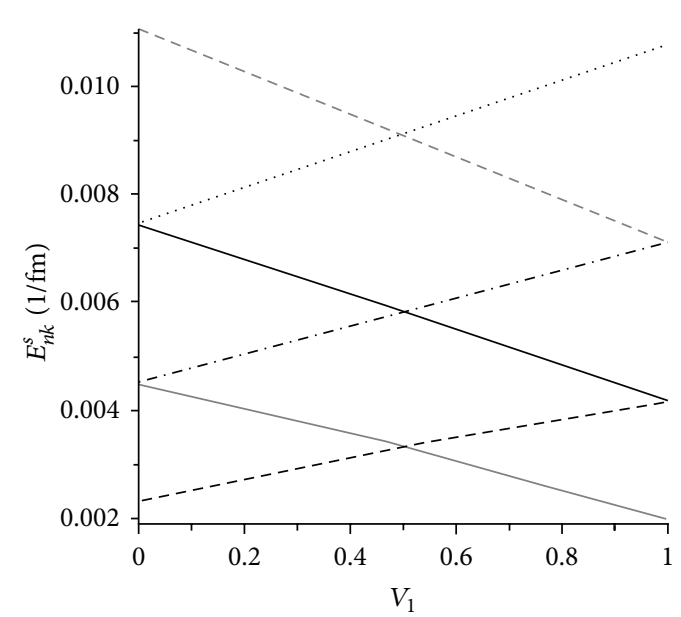

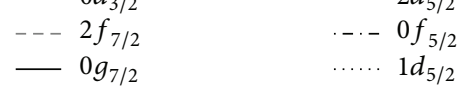

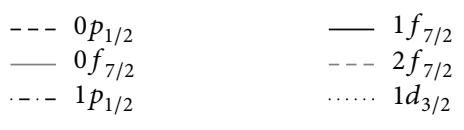

FIGURE 3: PSS: energy versus $V_{1}$ for pseudospin symmetry limit for $V_{0}=4 \mathrm{fm}^{-1}, H=0.5, \alpha=0.05 \mathrm{fm}, M=5 \mathrm{fm}^{-1}$, and $C_{\mathrm{ps}}=-5 \mathrm{fm}{ }^{-1}$. SS: energy versus $V_{1}$ for spin symmetry limit for $V_{0}=4 \mathrm{fm}^{-1}, H=0.5, \alpha=0.05 \mathrm{fm}, M=5 \mathrm{fm}^{-1}$, and $C_{s}=5 \mathrm{fm}^{-1}$.
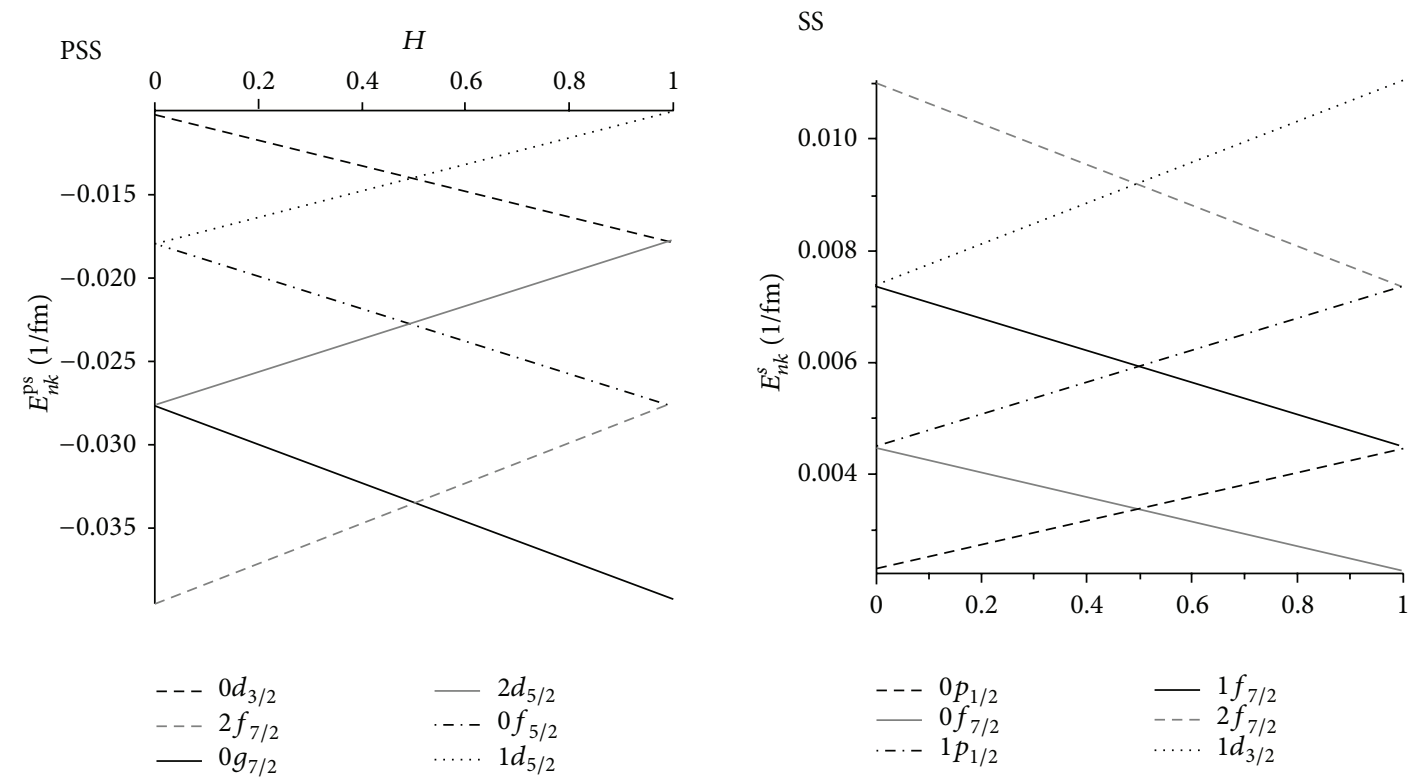

FIGURE 4: PSS: energy versus $H$ for pseudospin symmetry limit for $V_{0}=4 \mathrm{fm}^{-1}, V_{1}=0.5, \alpha=0.05 \mathrm{fm}, M=5 \mathrm{fm}^{-1}$, and $C_{\mathrm{ps}}=-5 \mathrm{fm}{ }^{-1}$. SS: energy versus $H$ for spin symmetry limit for $V_{0}=4 \mathrm{fm}^{-1}, V_{1}=0.5, \alpha=0.05 \mathrm{fm}, M=5 \mathrm{fm}^{-1}$, and $C_{s}=5 \mathrm{fm}^{-1}$.

where

$$
\begin{aligned}
\rho_{s}= & n^{2}+n+\frac{1}{2}+(2 n+1) \\
& \times \sqrt{\frac{1}{4}+\left(\kappa+V_{1}+H+1\right)\left(\kappa+V_{1}+H\right)} \\
& +\Lambda_{\kappa}\left(\Lambda_{\kappa}-1\right)+V_{1}\left(2 H+2 \kappa+\frac{3}{2}\right), \\
\lambda_{s}= & 2 n+1+2 \sqrt{\frac{1}{4}+\left(\kappa+V_{1}+H+1\right)\left(\kappa+V_{1}+H\right)},
\end{aligned}
$$

and the corresponding upper and lower radial wave functions are

$$
\begin{aligned}
F_{n, k}^{s}(r)= & e^{-2 \alpha \sqrt{f_{3}^{s}} r}\left(1-e^{-2 \alpha r}\right)^{(1 / 2)+\sqrt{(1 / 4)+f_{1}^{s}+f_{3}^{s}-f_{2}^{s}}} \\
& \times P_{n}^{\left(2 \sqrt{f_{3}^{s}}, 2 \sqrt{(1 / 4)+f_{1}^{s}+f_{3}^{s}-f_{2}^{s}}\right)}\left(1-2 e^{-2 \alpha r}\right), \\
G_{n, \kappa}^{s}(r)= & \frac{1}{M+E_{n \kappa}^{s}-C_{s}}\left(\frac{d}{d r}+\frac{\kappa}{r}-U(r)\right) F_{n \kappa}^{s}(r) .
\end{aligned}
$$



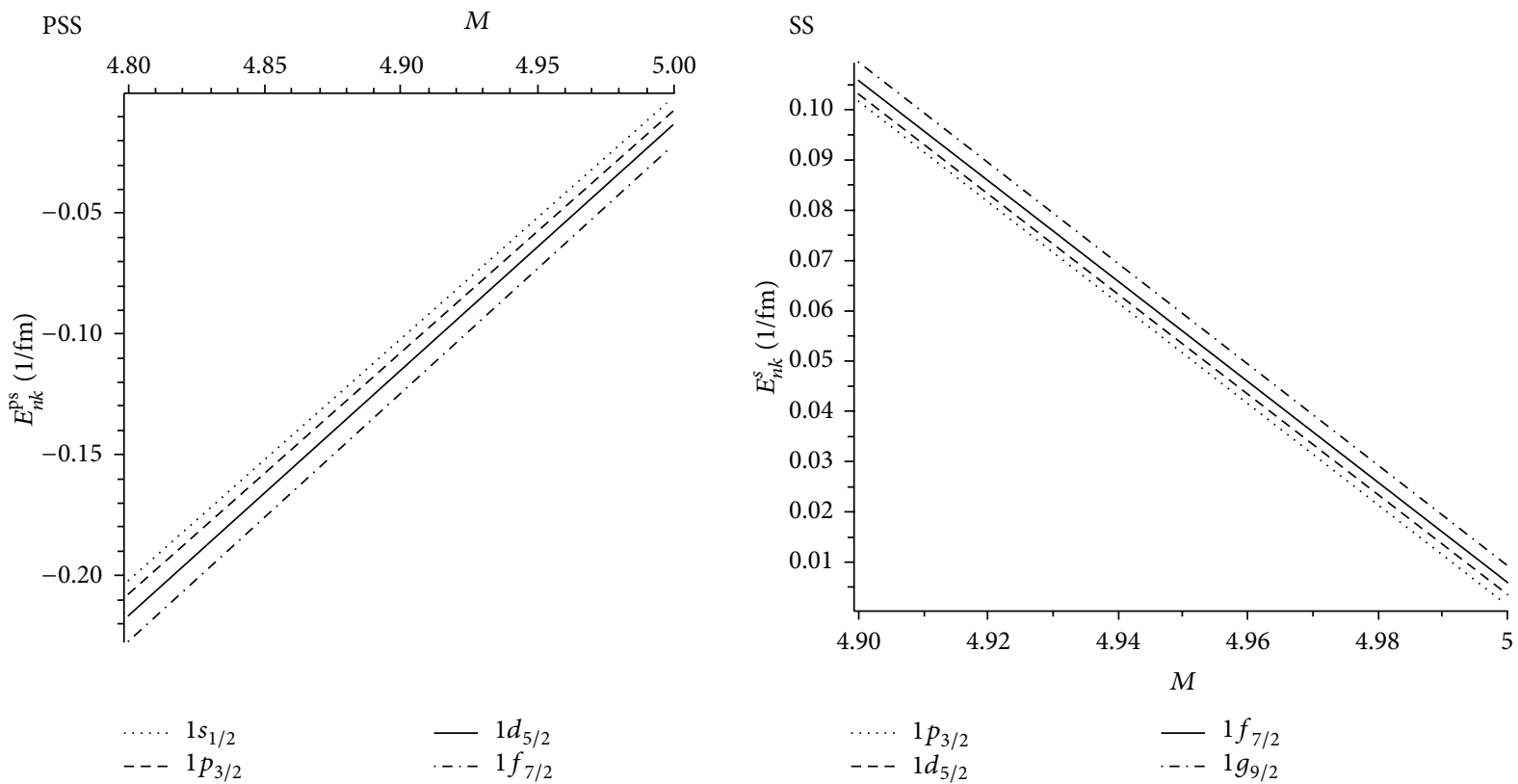

FIGURE 5: PSS: energy versus $M$ for pseudospin symmetry limit for $V_{0}=4 \mathrm{fm}^{-1}, V_{1}=0.5, H=0.5, \alpha=0.05 \mathrm{fm}$, and $C_{\mathrm{ps}}=-5 \mathrm{fm}{ }^{-1}$. SS: energy versus $M$ for spin symmetry limit for $V_{0}=4 \mathrm{fm}^{-1}, V_{1}=0.5, H=0.5, \alpha=0.05 \mathrm{fm}$, and $C_{s}=5 \mathrm{fm}^{-1}$.

PSS

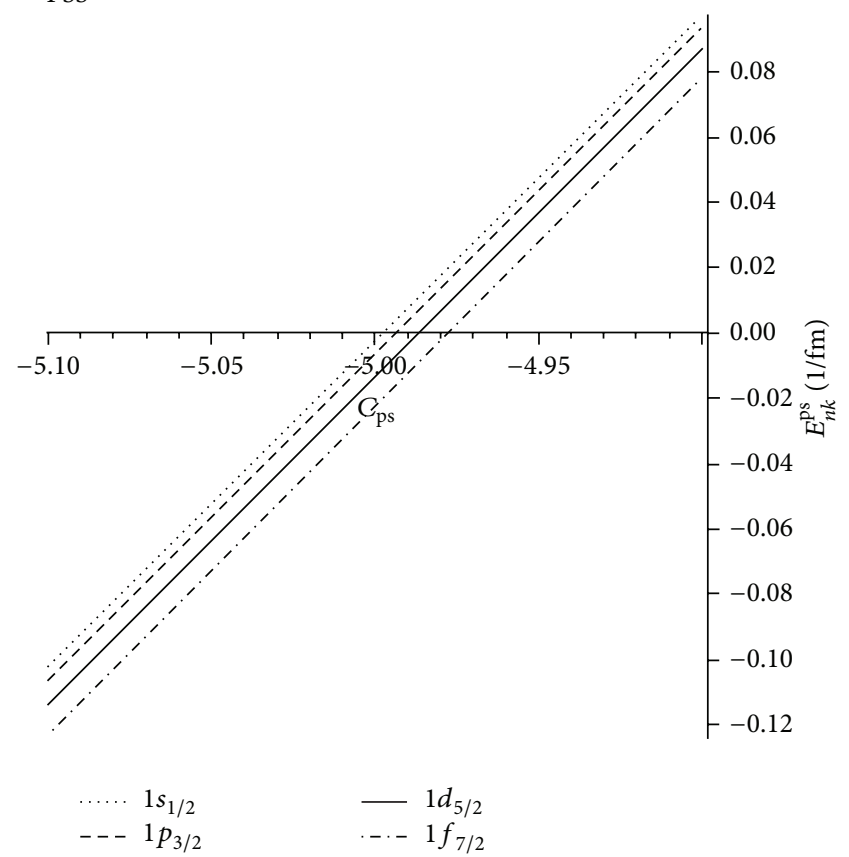

SS

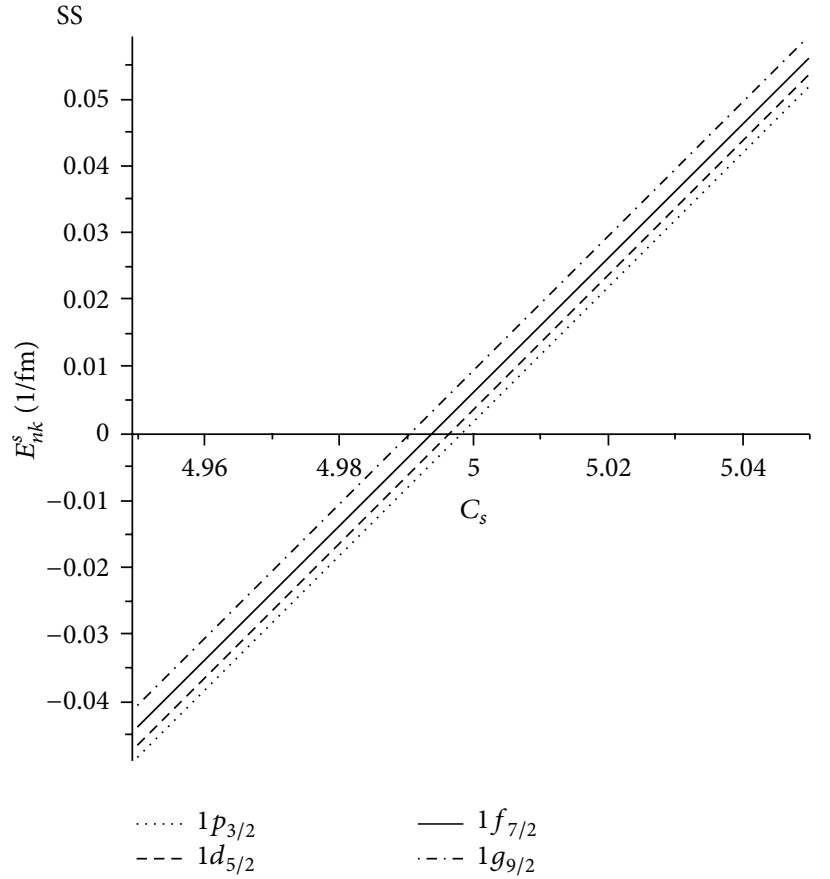

FIGURE 6: PSS: energy versus $C_{\mathrm{ps}}$ for pseudospin symmetry limit for $V_{0}=4 \mathrm{fm}^{-1}, V_{1}=0.5, H=0.5, \alpha=0.05 \mathrm{fm}$, and $M=5 \mathrm{fm}{ }^{-1}$. SS: energy versus $C_{s}$ for spin symmetry limit for $V_{0}=4 \mathrm{fm}^{-1}, V_{1}=0.5, H=0.5, \alpha=0.05 \mathrm{fm}$, and $M=5 \mathrm{fm}^{-1}$.

\section{Discussion and Numerical Results}

We obtained the energy eigenvalues in the absence $(H=0$, $\left.V_{1}=0\right)$ and the presence $\left(H=0.5, V_{1}=0.5\right)$ of the Coulomblike plus Yukawa tensor interaction for various values of the quantum numbers $n$ and $\kappa$. The results are reported in Tables 1 and 2 under the condition of the pseudospin and spin symmetries, respectively, where we can see the way the tensor interaction affects the degeneracy of the system. If we set $H=V_{1}=0$, the potential reduces into the Hulthén potential and our result is consistent with that of Aydoğdua et al. [27]. In Tables 1 and 2, we have compared our results with [27]. We represent the effects of the $\alpha$-parameter on the bound states for $H=V_{1}=0.5$ in Figure 2 . It is seen that if 

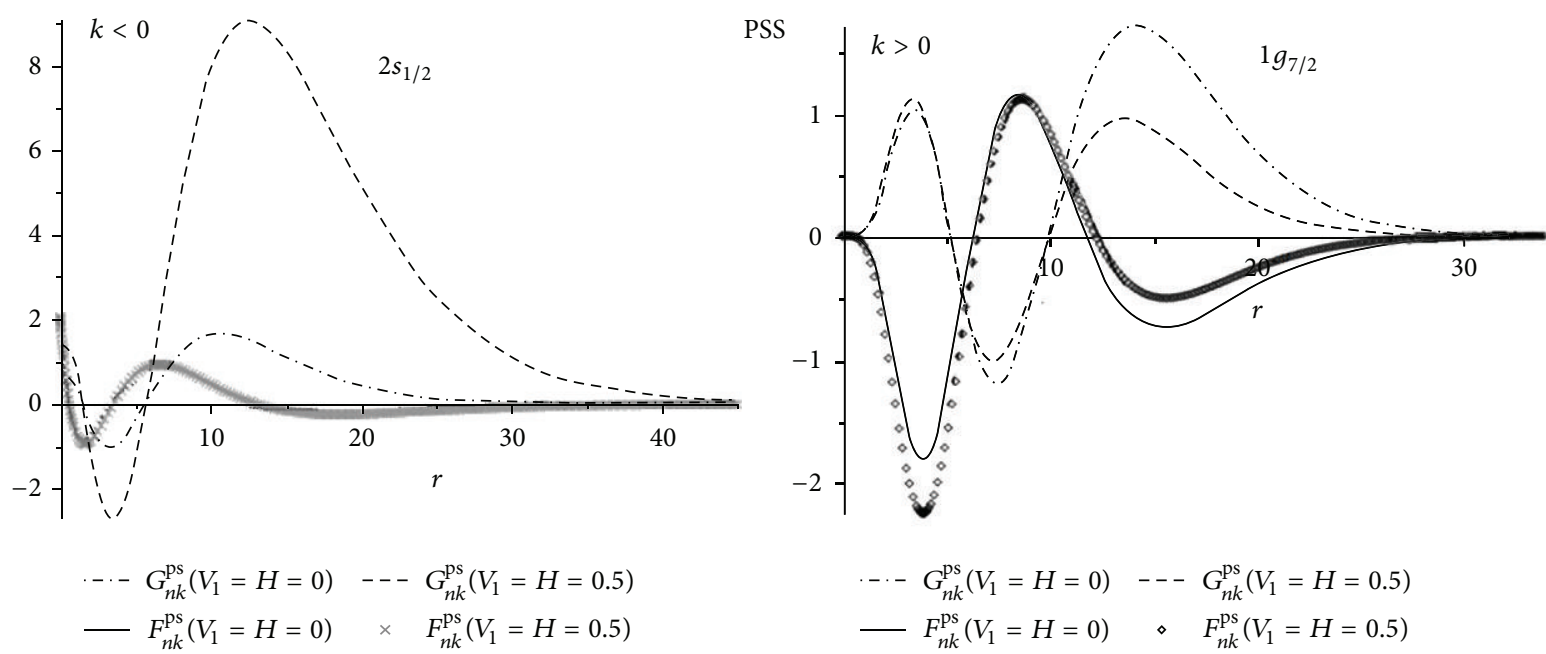

Figure 7: Wavefunction for pseudospin symmetry limit for $V_{0}=4 \mathrm{fm}^{-1}, M=5 \mathrm{fm}^{-1}, C_{\mathrm{ps}}=-5 \mathrm{fm}^{-1}$, and $\alpha=0.05 \mathrm{fm}$.
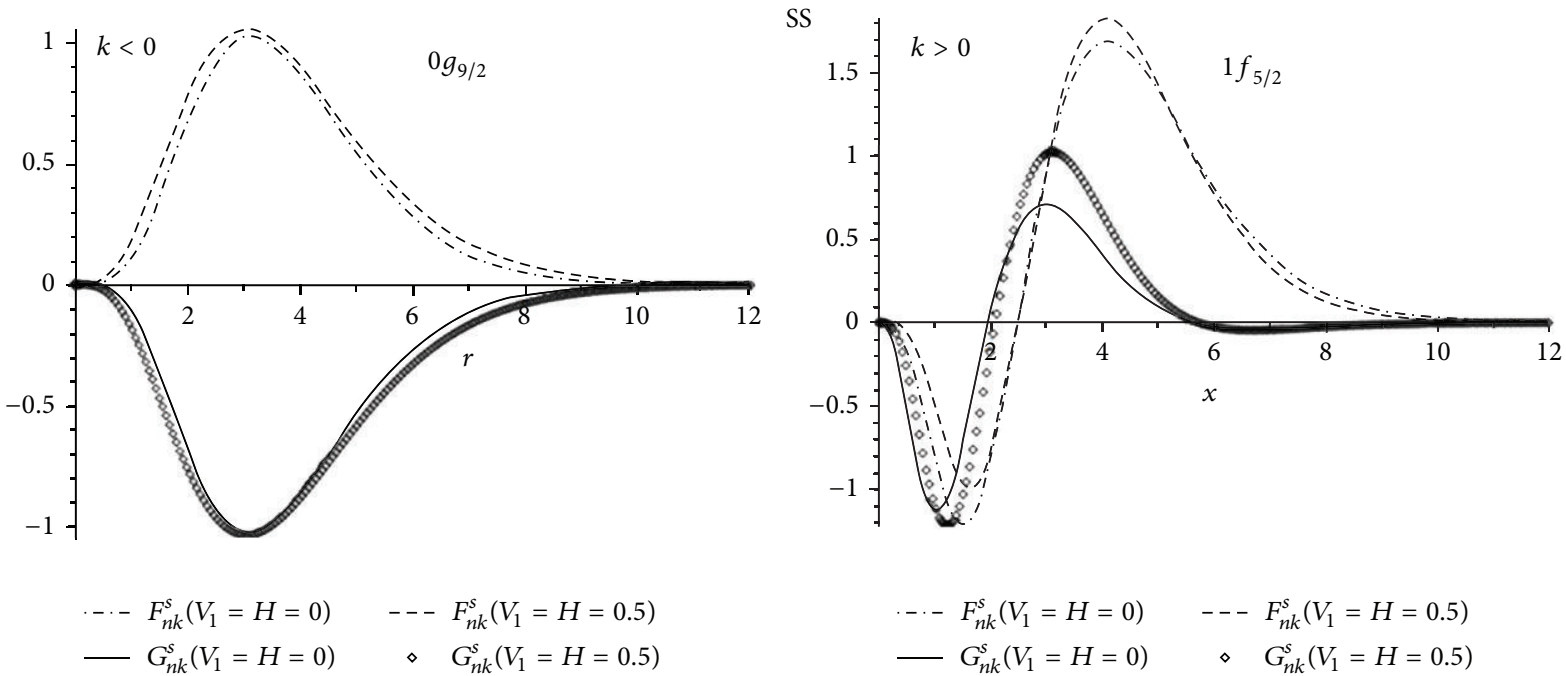

Figure 8: Wavefunction for spin symmetry limit for $V_{0}=4 \mathrm{fm}^{-1}, M=5 \mathrm{fm}^{-1}, C_{s}=5 \mathrm{fm}^{-1}$, and $\alpha=0.05 \mathrm{fm}$.

the $\alpha$-parameter increases, the bound states become more bounded in both for symmetry limits. Figures 3 and 4 present the effect of the tensor interaction on the bound states. We have plotted the energy versus $M$ in Figure 5. Figure 6 shows the effects of $C_{\mathrm{ps}}$ and $C_{s}$ on the boundstates. In Figures 7 and 8 , the wave functions are plotted for pseudospin and spin symmetry limits with and without a tensor interaction, respectively. It is seen in Figures 7 and 8 that the tensor interaction affects only the shape of the wave functions and does not change the node structure of the radial upper and lower components of the Dirac spinors.

\section{Conclusion}

In this paper, we obtained the approximate analytical solutions of the Dirac equation for the Hulthén potential with a novel generalized tensor interaction consisting of the
Coulomb and Yukawa interactions within the framework of pseudospin and spin symmetry limits using the NU technique. We have obtained the energy levels in a closed form and the corresponding wave functions in terms of the Jacobi polynomials. We also included some numerical results to investigate the way the combination of Coulomb and Yukawa potentials. Finally, the results of our work find many applications in both nuclear and Hadron physics and therefore provide more general solutions compared to other previous works performed in [30, 31].

\section{Appendix}

The NU method solves many linear second-order differential equations by reducing them to a generalized equation of hypergeometric type. Here, instead of the original formulation, we use the parametric version which enables us 
to solve a second-order differential equation of the form $[22,32]$

$$
\begin{aligned}
& \left\{\frac{d^{2}}{d s^{2}}+\frac{\alpha_{1}-\alpha_{2} s}{s\left(1-\alpha_{3} s\right)} \frac{d}{d s}\right. \\
& \left.\quad+\frac{1}{\left[s\left(1-\alpha_{3} s\right)\right]^{2}}\left[-\xi_{1} s^{2}+\xi_{2} s-\xi_{3}\right]\right\} \psi=0 .
\end{aligned}
$$

According to the NU method, the eigenfunction is

$$
\begin{aligned}
\psi(s)= & s^{\alpha_{12}}\left(1-\alpha_{3} s\right)^{-\alpha_{12}-\left(\alpha_{13} / \alpha_{3}\right)} \\
& \times P_{n}^{\left(\alpha_{10}-1,\left(\alpha_{11} / \alpha_{3}\right)-\alpha_{10}-1\right)}\left(1-2 \alpha_{3} s\right)
\end{aligned}
$$

and the energy of the system satisfies

$$
\begin{aligned}
& \alpha_{2} n-(2 n+1) \alpha_{5}+(2 n+1)\left(\sqrt{\alpha_{9}}+\alpha_{3} \sqrt{\alpha_{8}}\right) \\
& +n(n-1) \alpha_{3}+\alpha_{7}+2 \alpha_{3} \alpha_{8}+2 \sqrt{\alpha_{8} \alpha_{9}}=0
\end{aligned}
$$

where

$$
\begin{aligned}
& \alpha_{4}=\frac{1}{2}\left(1-\alpha_{1}\right), \quad \alpha_{5}=\frac{1}{2}\left(\alpha_{2}-2 \alpha_{3}\right), \\
& \alpha_{6}=\alpha_{5}^{2}+\xi_{1}, \quad \alpha_{7}=2 \alpha_{4} \alpha_{5}-\xi_{2}, \\
& \alpha_{8}=\alpha_{4}^{2}+\xi_{3}, \quad \alpha_{9}=\alpha_{3} \alpha_{7}+\alpha_{3}^{2} \alpha_{8}+\alpha_{6}, \\
& \alpha_{10}=\alpha_{1}+2 \alpha_{4}+2 \sqrt{\alpha_{8}}, \\
& \alpha_{11}=\alpha_{2}-2 \alpha_{5}+2\left(\sqrt{\alpha_{9}}+\alpha_{3} \sqrt{\alpha_{8}}\right), \\
& \alpha_{12}=\alpha_{4}+\sqrt{\alpha_{8}}, \quad \alpha_{13}=\alpha_{5}-\left(\sqrt{\alpha_{9}}+\alpha_{3} \sqrt{\alpha_{8}}\right) \\
& P_{n}^{(\alpha, \beta)}(x)=\frac{\Gamma(\alpha+n+1)}{n ! \Gamma(\alpha+\beta+n+1)} \\
& \quad \times \sum_{m=0}^{n}\left(\begin{array}{c}
n \\
m
\end{array}\right) \frac{\Gamma(\alpha+\beta+n+m+1)}{\Gamma(\alpha+m+1)}\left(\frac{x-1}{2}\right)^{m} .
\end{aligned}
$$

And $P_{n}^{(\alpha, \beta)}$ is Jacobi polynomial.

\section{Acknowledgment}

The authors wish to give their sincere gratitude to the referees for their careful and technical critiques.

\section{References}

[1] Y. Xu, S. He, and C. S. Jia, "Approximate analytical solutions of the Klein-Gordon equation with the Pöschl-Teller potential including the centrifugal term," Physica Scripta, vol. 81, Article ID 045001, 2010.

[2] X. Y. Gu and S. H. Dong, "Energy spectrum of the ManningRosen potential including centrifugal term solved by exact and proper quantization rules," Journal of Mathematical Chemistry, vol. 49, pp. 2053-2062, 2011.
[3] M. R. Pahlavani and S. A. Alavi, "Solutions of WoodsSaxon potential with Spin-Orbit and centrifugal terms through Nikiforov-Uvarov method," Communications in Theoretical Physics, vol. 58, p. 739, 2012.

[4] G. F. Wei and S. H. Dong, "A novel algebraic approach to spin symmetry for Dirac equation with scalar and vector second Pöschl-Teller potentials ," European Physical Journal A, vol. 43, pp. 185-190, 2010.

[5] C.-Y. Chen, D.-S. Sun, and F.-L. Lu, "The relativistic bound states of the Hartmann potentials," Physica Scripta, vol. 74, no. 4, article 001, pp. 405-409, 2006.

[6] C. S. Jia, X. P. Li, and L. H. Zhang, "Exact solutions of the KleinGordon equation with position-dependent mass for mixed vector and scalar kink-like potentials," Few-Body Systems, vol. 52, pp. 11-18, 2012.

[7] S. H. Dong and G. H. Sun, "The series solutions of the nonrelativistic equation with the Morse potential," Physics Letters A, vol. 314, pp. 261-266, 2003.

[8] G. F. Wei and S. H. Dong, "Pseudospin symmetry for modified Rosen-Morse potential including a Pekeris-type approximation to the pseudo-centrifugal term," European Physical Journal A, vol. 46, pp. 207-212, 2010.

[9] H. Hassanabadi, B. H. Yazarloo, S. Zarrinkamar, and A. A. Rajabi, "Duffin-Kemmer-Petiau equation under a scalar Coulomb interaction," Physical Review C, vol. 84, no. 6, Article ID 064003, 2011.

[10] J. N. Ginocchio, "Relativistic harmonic oscillator with spin symmetry," Physical Review C, vol. 69, no. 3, Article ID 034318, 2004.

[11] J. N. Ginocchio, "Pseudospin as a relativistic symmetry," Physical Review Letters, vol. 78, pp. 436-439, 1997.

[12] P. R. Page, T. Goldman, and J. N. Ginocchio, "Relativistic symmetry suppresses quark spin-orbit splitting," Physical Review Letters, vol. 66, pp. 204-207, 2001.

[13] J. N. Ginocchio, "Relativistic symmetries in nuclei and hadrons," Physics Reports, vol. 414, no. 4-5, pp. 165-261, 2005.

[14] J. N. Ginocchio, A. Leviatan, J. Meng, and S.-G. Zhou, "Test of pseudospin symmetry in deformed nuclei," Physical Review C, vol. 69, no. 3, Article ID 034303, 2004.

[15] F. Taskin, "Approximate solutions of the Dirac equation for the Manning-Rosen potential including the spin-orbit coupling term," International Journal of Theoretical Physics, vol. 48, pp. 1142-1149, 2000.

[16] C. S. Jia, P. Guo, and X. L. Peng, "Exact solution of the Dirac-Eckart problem with spin and pseudospin symmetry," International Journal of Theoretical Physics, vol. 39, p. 7737, 2006.

[17] H. Hassanabadi, E. Maghsoodi, S. Zarrinkamar, and H. Rahimov, "Approximate any l-state solutions of Dirac equation for modified deformed Hylleraas potential by the NikiforovUvarov method," Journal of Mathematical Physics, vol. 21, no. 2, Article ID 120302, 2012.

[18] E. Maghsoodi, H. Hassanabadi, and S. Zarrinkamar, "Spectrum of Dirac Equation under Deng-Fan scalar and vector potentials and a Coulomb tensor interaction by SUSYQM," Few-Body Systems, vol. 53, pp. 528-538, 2012.

[19] E. Maghsoodi, H. Hassanabadi, H. Rahimov, and S. Zarrinkamar, "Arbitrary-state solutions of the Dirac equation for a Möbius square potential using the Nikiforov-Uvarov method," Chinese Physics C, vol. 37, Article ID 04105, 2013.

[20] H. Hassanabadi, E. Maghsoodi, and S. Zarrinkamar, "Relativistic symmetries of Dirac equation and the Tietz potential," European Physical Journal Plus, vol. 127, no. 3, pp. 1-14, 2012. 
[21] A. N. Ikot, "Solutions of Dirac Equation for generalized hyperbolical potential including Coulomb-Like tensor potential with spin symmetry," Few-Body Systems, vol. 53, pp. 549-555, 2012.

[22] A. F. Nikiforov and V. B. Uvarov, Special Functions of Mathematical Physics, Birkhauser, Basel, Switzerland, 1988.

[23] F. Cooper, A. Khare, and U. Sukhatme, "Supersymmetry and quantum mechanics," Physics Report, vol. 251, no. 5-6, pp. 267385, 1995.

[24] A. N. Ikot, E. Maghsoodi, S. Zarrinkamar, and H. Hassanabadi, "Relativistic spin and pseudospin symmetries of inversely quadratic Yukawa-like plus Mobius Square potentials including a Coulomb-like tensor interaction," Few-Body Systems, 2013.

[25] M. Hamzavi, S. M. Ikhdair, and B. I. Ita, "Approximate spin and pseudospin solutions to the Dirac equation for the inversely quadratic Yukawa potential and tensor interaction," Physica Scripta, vol. 85, no. 4, Article ID 045009, 2012.

[26] H. Hassanabadi, E. Maghsoodi, and S. Zarrinkamar, "Spin and pseudospin symmetries of Dirac Equation and the Yukawa potential as the tensor interaction," Communications in Theoretical Physics, vol. 58, p. 807, 2012.

[27] O. Aydoğdua, E. Maghsoodi, and H. Hassanabadi, "Dirac equation for the Hulthén potential within the Yukawa-type tensor interaction," Chinese Physics B, vol. 22, Article ID 010302, 2013.

[28] H. Hassanabadi, S. Zarrinkamar, and H. Rahimov, "Approximate solution of D-dimensional Klein-Gordon equation with Hulthen-type potential via SUSYQM," Communications in Theoretical Physics, vol. 56, pp. 423-428, 2011.

[29] C. L. Pekeris, "The rotation-vibration coupling in diatomic molecules," Physical Review, vol. 45, no. 2, pp. 98-103, 1934.

[30] H. Hassanabadi, E. Maghsoodi, A. N. Ikot, and S. Zarrinkamar, "Dirac equation under Manning-Rosen potential and Hulthén tensor interaction," European Physical Journal Plus, vol. 128, article 79, 2013.

[31] Y. Sun, S. He, and C. S. Jia, "Equivalence of the deformed modified Rosen-Morse potential energy model and the Tietz potential energy model," Physica Scripta, vol. 87, Article ID 025301, 2013.

[32] C. Tezcan and R. Sever, "A general approach for the exact solution of the Schrödinger equation," International Journal of Theoretical Physics, vol. 48, pp. 337-350, 2009. 

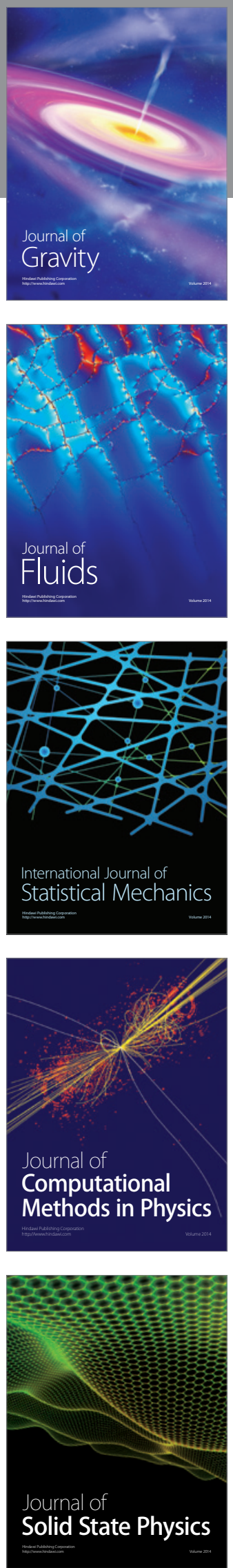

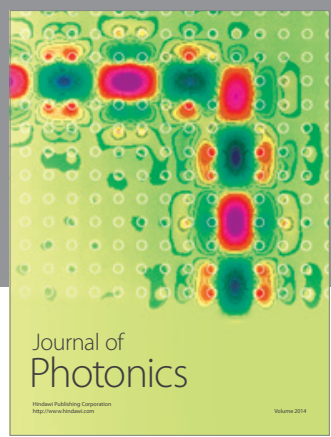

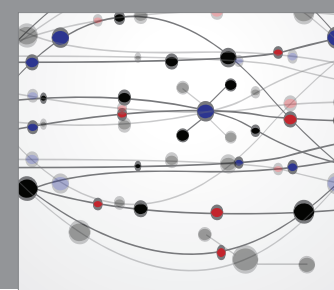

The Scientific World Journal

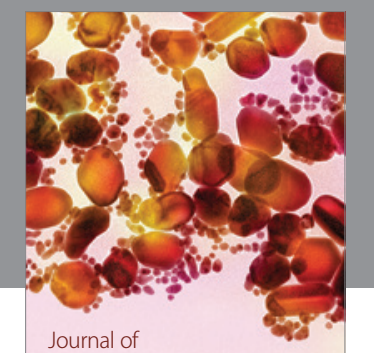

Soft Matter
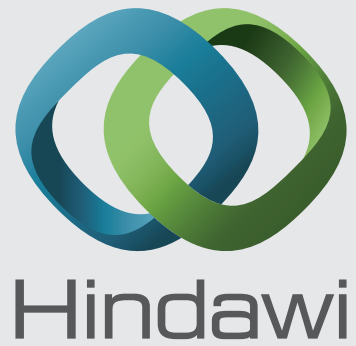

Submit your manuscripts at

http://www.hindawi.com
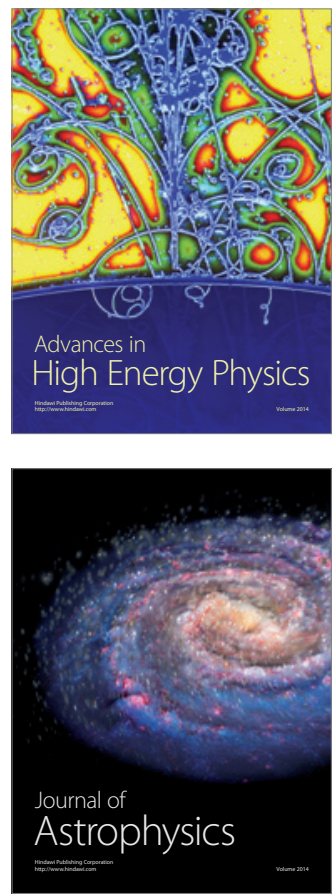
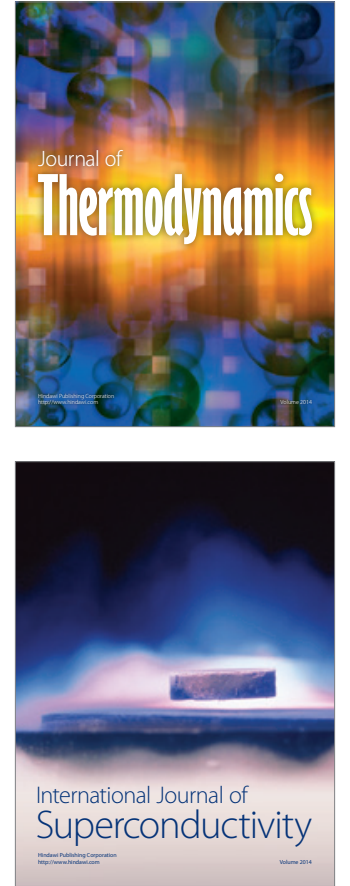
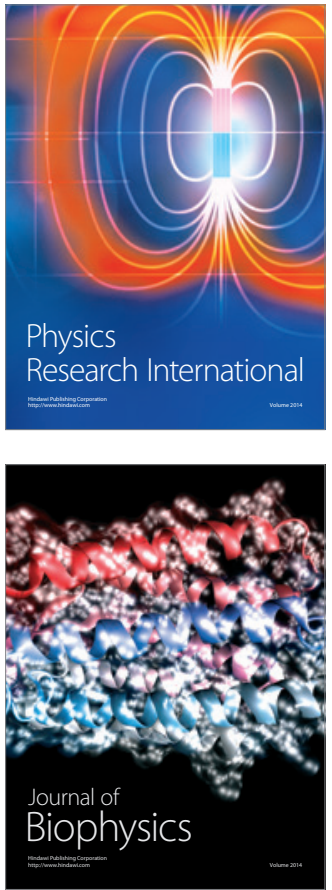
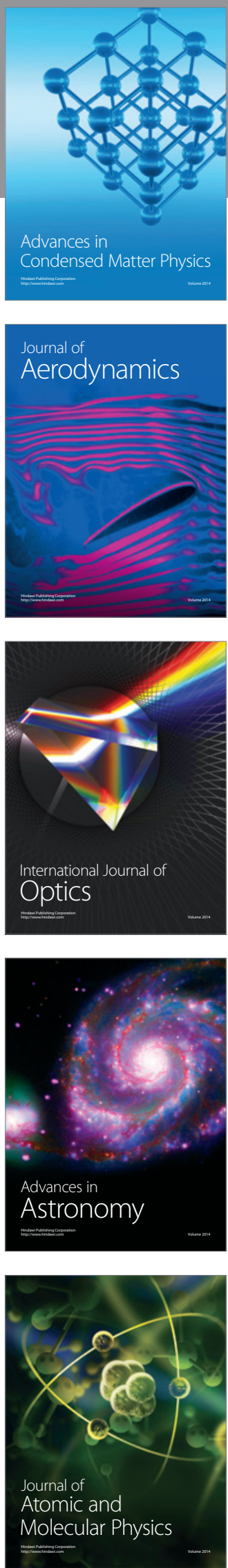